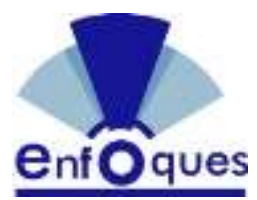

Enfoques. Revista de Investigación en Ciencias de la Administración http://doi.org/10.33996/revistaenfoques.v3i11.67

No. 11| Volumen 3 | Julio - Septiembre 2019 http://revistaenfoques.org ISSN: $2616-8219$

pp. $217-234$

\section{ANSIEDAD Y ESTRATEGIAS DE AFRONTAMIENTO EN TRABAJADORES PETROLEROS}

\author{
ANXIETY AND STRATEGIES OF AFRONTATION IN OIL WORKERS
}

\author{
Jesús Villasmil
}

Artículo recibido marzo 2019 | Arbitrado abril 2019 | Publicado 01 de julio 2019

\section{Resumen}

La presente investigación estuvo dirigida a establecer la relación existente entre la ansiedad y las estrategias de afrontamiento de los trabajadores de la empresa EHCOPEK-S.A. ubicada en el estado Zulia. En cuanto al marco metodológico se trató de un estudio de naturaleza cuantitativa cuya base epistemológica es el positivismo apoyado en una investigación de carácter confirmatorio con diseño de campo y de tipo transversal. Para el Inventario (CSI) se obtuvo puntajes altos $(0,914)$ para la escala global, lo que demuestra que el instrumento posee longitud y 0.63 a 0.86 , lo cual señala que el inventario es homogéneo con respecto al constructo medido y sus puntajes son consistentes dentro de la muestra. Se concluyó que las variables medidas no cuentan con una correlación significativa, de igual manera se evidenciaron niveles de ansiedad media así como que una mayoría significativa de la muestra encuestada emplea estrategias orientadas a la resolución del problema lo cual indica que existe un manejo adecuado de situaciones amenazantes.

Palabras clave: Ansiedad; estrategias de afrontamiento; industria petrolera

\begin{abstract}
This research was aimed at establishing the relationship between anxiety and coping strategies for workers of the company EHCOPEK-S.A. located in the state Zulia. Regarding the methodological framework, it was a quantitative study whose epistemological basis is positivism supported by a confirmatory investigation with a field and cross-sectional design. For the Inventory (CSI), high scores (0.914) were obtained for the global scale, which shows that the instrument has length and 0.63 to 0.86 , which indicates that the inventory is homogeneous with respect to the measured construct and its scores are consistent within of the sample. It was concluded that the measured variables do not have a significant correlation, in the same way levels of average anxiety were evidenced as well as that a significant majority of the sample surveyed employs strategies aimed at solving the problem which indicates that there is an adequate management of situations threatening.
\end{abstract}

Key works: Anxiety; coping strategies; oil industry

\section{Jesús Villasmil}

jvillasmils@gmail.com

EHCOPEK-S.A. y Universidad del Zulia, Venezuela

Ingeniero Mecánico, egresado de la Universidad del Zulia, Venezuela. Psicólogo Universidad de Yacambú. Maestría en Gerencia de Proyectos Industriales, Universidad Rafael Belloso Chacín, Venezuela 


\section{INTRODUCCIÓN}

Los constantes cambios políticos y económicos actuales del mundo laboral repercuten en grandes cambios en el entorno de las organizaciones y en especial en aquellas cuyo nicho de mercado es la industria energética. Las fluctuaciones económicas mundiales y las diferentes situaciones sociopolíticas entre otras, afectan la estabilidad laboral de los trabajadores y generan incertidumbre en cuanto al futuro laboral, lo cual activa la necesidad de enfrentar nuevos retos que sobrepasan la capacidad de respuesta de una persona y esta circunstancia a su vez, repercute en la salud física y mental.

En un mundo globalizado como el actual, es de vital importancia hallar mecanismos para afrontar de la mejor manera las situaciones ansiógenas que se presentan en el día a día. De allí la relevancia de estudiar las características personales de los empleados de una organización, para tener una visión general de su desarrollo y comportamiento y de esta manera crear espacios de mejoras donde no solo conocer qué aspectos contribuyen con el bienestar psicológico del empleado y cuáles son sus fuentes de insatisfacción, sino también canalizar sus inquietudes para ayudarles a desarrollar maneras de afrontarlas, lo cual redunda en beneficio del binomio trabajador-organización.

En efecto, el Consejo Europeo del Cerebro en el año 2011, expuso que los trastornos de mayor incidencia en el ámbito laboral son el estrés con un $25 \%$, seguido de la ansiedad con un $14 \%$, lo cual refleja que entre un $50 \%$ y $60 \%$ de las bajas laborales están relacionadas con dichos trastornos; estos datos son corroborados por la Sociedad Española de Medicina Psicosomática (2016) cuando explica un repunte del $12 \%$ de casos de síndromes ansioso-depresivos cuyos factores de origen se encuentran, como una de las principales causas, en las condiciones sociolaborales.

Al mismo tiempo, la institución mencionada alerta que con el tiempo dichos trastornos pueden causar hipertensión, somnolencia, problemas de estómago y problemas musculares, como lumbalgias $y$ contracturas.

Al respecto, la Organización Internacional del Trabajo (OIT) en su informe del año 2016 expone que la reciente crisis económica mundial ha conllevado a numerosas empresas a reducir su capacidad operativa para mantener la competitividad, pero dichos procesos de reestructuración ocasionan en el trabajador incertidumbre sobre su futuro y hostilidad en el ambiente de trabajo por cómo se vislumbra el panorama de la empresa, aunado a esto y tratando de dar ayuda de alguna manera muchas empresas ofrecen flexibilidad en los horarios por lo que el trabajador asume cargas de trabajo mayores a las regulares, para poder contar con fuentes de ingreso alternas para satisfacer sus necesidades, lo cual origina disminución de la atención en la gestión de los riesgos, lo que se convierte en posibilidades de accidentes laborales, por distracciones lo cual compromete la salud ocupacional.

Sobre lo anteriormente citado, esta misma organización en el año 2008 expuso que en América Latina no se han dado suficientes investigaciones sobre la ansiedad laboral, con la finalidad de identificar los niveles de la misma según la cultura del trabajador y las diferencias conductuales asociadas según la nacionalidad. Esta información ya fue descrita por Cooper y Payne (1992) quienes destacan que los problemas de ansiedad laboral parecen existir en todo el mundo, pero son pocas las estadísticas que documentan su naturaleza y 
extensión fuera de Estados Unidos, por el contrario, en otros países este tipo de trastornos son registrados como padecimientos físicos, pero indagando sobre el origen de los mismos los datos obtenidos indican que están estrechamente ligados a situaciones laborales causantes de ansiedad.

Ahora bien, el panorama descrito sobre la incidencia de la ansiedad en el sector laboral conduce a los trabajadores a desarrollar maneras de afrontarla que en muchos casos no son las adecuadas para tal fin. En este sentido, Charry (2012) expone que para el empleo de una determinada estrategia de afrontamiento se debe tener en cuenta el contexto en el que se encuentra el individuo, pues si bien es cierto, que muchas estrategias pueden llegar a ser beneficiosas para algunas personas en ciertas situaciones, las mismas pudieran ser perjudiciales para otras personas en otro tipo de situaciones.

En relación a lo expuesto, la OMS (2008) estima que a nivel mundial sólo entre el 5 y $10 \%$ de los trabajadores en los países en desarrollo y entre el 20 y $50 \%$ de los trabajadores en países industrializados (con pocas excepciones) tienen acceso a servicios de salud ocupacional adecuados; aunado a esto se tienen escasos datos sobre enfermedades asociadas a factores psicosociales.

En este sentido, es importante destacar que las diversas situaciones que generan ansiedad, estrés o incertidumbre en los trabajadores de cualquier organización, generan impacto en la calidad de vida. Se presentan alteraciones fisiológicas y conductuales que desencadenan patologías que afectan no solo la salud del trabajador, sino que también representan para la organización incremento de los costos de inversión en salud laboral, ineficiencia en el uso de los recursos, y aumento de los índices de accidentabilidad.
En este mismo orden de ideas, Kort, García y Pérez (1998) realizaron una investigación sobre el estado psicológico de los ciudadanos en Caracas para el cual diseñaron un cuestionario con indicadores de ansiedad, depresión e ira aplicado a 1803 participantes, divididos en clase media alta y media baja. Este trabajo arrojó como resultado que los grupos examinados sin importar el nivel de ingresos económicos presentaron un alto grado de ansiedad, destacándose conductas de resignación, pasividad y desesperanza, así como también se pudo evidenciar distorsión cognitiva pues los sujetos encuestados aseguraban sentirse felices.

Los resultados obtenidos en dicho estudio condujeron a los autores a concluir que los ciudadanos encuestados no contaban con suficientes destrezas para hacer frente a situaciones ansiógenas.

En cuanto a la situación objeto de estudio de esta investigación, es necesario resaltar que desde el descubrimiento del petróleo como fuente de energía con impacto en el desarrollo industrial, es de conocimiento público el dinamismo de este tipo de industria y los aspectos que condicionan su operatividad.

Las demandas del mercado internacional, en cuanto a reducciones de producción y costos de la cesta petrolera, así como los cambios políticos que se suscitan a nivel mundial, obliga a muchas empresas del sector a reducir al mínimo sus operaciones para poder mantenerse activas en una economía cambiante.

En efecto, esta realidad producto de decisiones macroeconómicas que involucran las actividades de la industria petrolera, afecta a sus trabajadores con agentes ansiógenos que tienen repercusión en el bienestar psicológico, y les moviliza al desarrollo de maneras de afrontar este tipo 
de situaciones, con el objetivo de obviar sus consecuencias sobre su salud y en sus entornos familiares.

Debe señalarse que por lo general las actividades de la industria petrolera requieren de alta precisión y concentración lo que involucra el ámbito cognitivo. Desde el punto de vista fisiológico, la ansiedad altera diversas funciones básicas tales como los patrones del sueño, el ritmo cardiaco, rigidez muscular lo que provoca un deterioro funcional del trabajador que puede generar consecuencias negativas, por lo que resulta claro, que el trabajador sea objeto de estudio en cuanto a los niveles de ansiedad que experimenta en su entorno laboral, ya que la existencia de niveles de ansiedad en dichos trabajadores, repercute no solo en su desempeño personal, sino que afecta de manera inmediata al equipo de trabajo y pone en riesgo al trabajador $y$ a sus compañeros.

Actualmente, en vista de la inestabilidad del mercado petrolero venezolano, los trabajadores de este sector, ubicados en el estado Zulia, no están exentos de la realidad descrita anteriormente. Las fluctuaciones de orden laboral, económico y político les afectan de igual manera, tal es el caso de la contratista EHCOPEK-S.A., cuyo nicho de mercado se centra principalmente en actividades de ingeniería y construcción en instalaciones petroleras ubicadas en el lago de Maracaibo, donde los trabajadores se han visto involucrados en situaciones que afectan directamente su bienestar psicológico por lo que regularmente presentan recurrentes estados de ansiedad que les obliga a desarrollar y aplicar un conjunto de habilidades y destrezas para afrontarlas y lograr así adaptarse, conservar la integridad psicológica y mantener su funcionalidad como individuo.
La problemática antes planteada evidencia la importancia de indagar sobre la ansiedad existente en las personas objeto de este estudio, al igual que sobre las estrategias de afrontamiento utilizadas en función del contexto señalado, con el propósito de dar respuesta a la interrogante de investigación que el tema genera: ¿Cuál es el grado de relación existente entre la ansiedad y las estrategias de afrontamiento en los trabajadores petroleros de la empresa EHCOPEK-S.A. ubicada en el estado Zulia.?

\section{Fundamentación teórica}

En las últimas décadas el análisis de la ansiedad en el mundo de la psicología es un tema de gran relevancia, los cambios vertiginosos en la sociedad actual obliga a los especialistas en la materia a desarrollar competencias que permitan el tratamiento clínico de la misma.

El mundo actual, requiere un mayor grado de flexibilidad, seguridad en sí mismo, capacidad de iniciativa y capacidad de respuesta ante el entorno, puesto que son muchas las contrariedades y cambios que se viven con un gran dinamismo y para los cuales se requiere contar con herramientas cognitivas, conductuales y emocionales para responder de manera acorde al propósito de adaptarse al contexto en que se vive.

En ese sentido, para Cano (1986) la ansiedad es un manera de responder ante una situación, la cual contiene elementos cognitivos no gratos y que por lo tanto conllevan tensión y aprensión; elementos fisiológicos con alta capacidad para activar el sistema nervioso autónomo y aspectos motores que generalmente tienen que ver con comportamientos poco ajustados $y$ escasamente adaptativos. 
En ese orden de ideas, Lluch, Novel y Ortiz (2005) explican que la ansiedad puede clasificarse según su intensidad en cuatro niveles: leve, moderado, grave y de pánico y que cada uno de estos niveles posee sus manifestaciones características como se describen a continuación:

Ansiedad leve: su principal característica es el estado de alerta, la persona que la padece se encuentra en condiciones de afrontar y resolver problemas, sin embargo los síntomas neurológicos presentes son el insomnio, sensación de malestar y agotamiento físico.

Ansiedad moderada: afecta la atención, la precepción y genera dificulta de concentración. Desde el punto de vista fisiológico se presenta aumento de la frecuencia cardiaca o respiratoria, temblores y estremecimientos. Hay afectación del sistema sensoperceptivo y osteomuscular; desde el punto de vista psicológico se presenta desasosiego, inquietud interior, inseguridad, temor a perder el control, trastornos de memoria, pensamientos negativos, dificultad en las relaciones interpersonales, entre otros.

Ansiedad grave: su principal síntoma es la dificultad para concentrase, trastornos psicosomáticos y visión cerrada de la realidad. Desde el punto de vista fisiológico la persona padece taquicardia, migraña, náuseas, hipertensión, trastornos digestivos, trastornos sexuales, entre otros.

Ansiedad de pánico: según el DSM-5 (2014), ésta se caracteriza por una percepción distorsionada de la realidad con incapacidad de comunicarse con fuertes alteraciones del equilibrio orgánico que pueden llegar a comprometer la salud mental.

En función de lo expuesto por los autores, se puede observar que la ansiedad puede afectar significativamente la calidad de vida de las personas, el hecho de que las relaciones interpersonales sean influenciadas por alteraciones cognitivas y conductuales de la persona ansiosa compromete la actividad socializadora del individuo, repercutiendo en su desempeño laboral, en las relaciones familiares y la funcionalidad misma de la persona por ausencia del bienestar psicológico.

Por lo anteriormente expuesto, puede decirse que el bienestar psíquico de un trabajador es esencial para un buen desempeño laboral, y que se debe abordar de manera multifactorial, estableciendo un marco en el cual tiene lugar un proceso efectivo de envío y recepción de información, en un acto donde se posibilita el intercambio de opiniones, sentimientos, creencias dentro de una empresa, o lugar de trabajo.

Asimismo, Spielberg (1972), define la ansiedad como un estado emocional inmediato y modificable en el tiempo, caracterizado por una combinación única de sentimientos de tensión, aprensión, nerviosismo, pensamientos molestos y cambios fisiológicos. Cabe destacar que la ansiedad estado no se manifiesta directamente en la conducta y debe ser inferida por la frecuencia con la que un individuo experimenta aumentos en sus estados de ansiedad. Los niveles altos de la misma son apreciados como molestos y obligaran al individuo a poner en marcha habilidades que le permitan afrontar la situación causante de la aparición de la misma.

En relación a lo descrito por el autor, la ansiedad estada solo surge ante eventos específicos y por lo tanto no está asociada a los patrones de personalidad, sin embargo, la presencia de la misma en altos niveles desencadena respuestas defensivas cuyas manifestaciones se dan en el orden 
cognitivo, conductual y fisiológico evidenciándose a través de cambios temporales en la persona que pudieran ser dañinos.

Es así como en el ámbito laboral, cuando un trabajador experimenta un alto nivel de ansiedad estado en su entorno, se expone a una alta probabilidad de ocurrencia de un evento no deseado como es el caso de la industria petrolera, objeto de este estudio, donde es común el manejo de máquinas o herramientas de alta peligrosidad cuyos operadores deben encontrarse en óptimas condiciones físicas, mentales y emocionales para evitar situaciones que pueden afectar el desempeño laboral del trabajador, del equipo e incluso de la organización.

En cuanto a la ansiedad como rasgo, Spielberg (1972), explica que la ansiedad rasgo hace referencia a las diferencias individuales de ansiedad relativamente estables, siendo éstas una disposición, tendencia o rasgo, por lo tanto, aquello sujetos con alto grado de ansiedad rasgo son más propensos a experimentar alto grado de ansiedad estado, de manera más frecuente; en otras palabras, la ansiedad rasgo se encuentra inmersa dentro de las características de la personalidad y por lo tanto este tipo de individuos ven con mayor peligrosidad aquellas situaciones que le resulten amenazantes por lo que desarrolla estrategias de afrontamiento más rápidas y más agresivas.

En referencia a lo expresado por el autor, se infiere que la ansiedad rasgo constituye un factor de la personalidad por lo que los altos niveles de la misma conllevan a maximizar la magnitud de las amenazas que surgen, en tal sentido, las manifestaciones cognitivas, conductuales y fisiológicas de la ansiedad se presentan con mayor frecuencia afectando la calidad de vida de la persona.
En tal sentido, cuando la medición efectuada a un trabajador arroja un alto nivel de ansiedad estado, aumenta su probabilidad de desarrollar patologías psicológicas $u$ orgánicas. En el caso específico de los trabajadores objeto de estudio de esta investigación, es importante ya que como se mencionó anteriormente el ambiente laboral referido al contexto petrolero se caracteriza por tener elementos estresores como son las altas cargas de trabajo, los horarios extendidos más allá de lo reglamentado, entre otros, así como la existencia de incertidumbre laboral debido a fluctuaciones en el mercado por la situación política y económica del país.

En relación con las manifestaciones de la ansiedad, Navarrete (2013), menciona que la ansiedad es producida por medio de uno o varios estímulos, sin que la persona se percate de ellos. Se refleja en la apariencia de la persona por lo que se consideran tres aspectos fundamentales que son los síntomas físicos, los síntomas cognitivos y los síntomas conductuales, sin embargo, estas manifestaciones no siempre ocurren de manera simultánea y tampoco se activan de la misma manera en todas las personas, aunque todos comparten el núcleo central de la ansiedad como síntoma fundamental.

Con base en lo expuesto por el autor, se entiende que si bien la ansiedad es una experiencia natural y subjetiva en los seres humanos, la misma también tiene implicaciones de orden físico, es decir, puede ser detectada por signos y síntomas observables, por lo tanto su relación con la biología de la persona está plenamente comprobada, en otras palabras, la ansiedad surge desde un estado mental y se manifiesta aguas abajo en un estado corporal como una preparación para amenazas futuras sean reales o no. 
Es así como, en el caso objeto de estudio la presencia de niveles anormales de ansiedad está asociada a la de la incertidumbre de no poder proyectar un futuro estable, o a la inquietud por conocer que va a pasar cuando merme la cantidad de trabajo a ejecutar debido a lo fluctuante del mercado, o a la severidad de la situación económica dictada por situaciones políticas. Las circunstancias descritas anteriormente hacen que el futuro sea visto como una amenaza lo que activa los mecanismos de alerta que en muchos casos compromete la estabilidad emocional de los trabajadores y afecta su calidad de vida, la motivación en el trabajo y la productividad.

Desde el punto de vista cognitivo y con base en lo expuesto por Navarrete (2013) cognitivamente la ansiedad puede manifestarse en sentimientos de malestar, preocupación, hipervigilancia, tensión, miedo, inseguridad, sensación de pérdida de control, dificultad para decidir, presencia de pensamientos negativos sobre uno mismo, pensamientos negativos de como la persona actúa ante los demás, temor a que los demás perciban las debilidades propias, dificultades para estudiar, pensar o concentrarse, percepción de fuertes cambios fisiológicos, entre otros.

Tal como se mencionó, la ansiedad se origina como una experiencia subjetiva en la mente de la persona, y es desde ese punto de partida, la mente, desde donde surgen las desarmonías en el ser humano. La sintomatología de la ansiedad en muchos casos compromete el manejo equilibrado de situaciones de la vida cotidiana, y con mayor razón cuando el medio es inestable como muchas veces suele suceder en los ambientes laborales. El manejo no adecuado de la ansiedad desde el punto de vista cognitivo traerá como consecuencias afectaciones de orden orgánico que pueden trascender en la calidad de las relaciones interpersonales y el desempeño laboral inadecuado lo que aumenta la probabilidad de ocurrencia de accidentes e insatisfacción laboral.

En cuanto a las manifestaciones fisiológicas de la ansiedad, Medrano y Mirantes (2008), explican que la ansiedad se manifiesta a través de la activación de diferentes sistemas, principalmente el sistema nervioso autónomo y el sistema nervioso motor, aunque también se pueden presentar activaciones del sistema inmune y sistema endocrino. De todos los cambios que ocurren la persona solo percibe algunos tales como, aumento de frecuencia cardiaca, aumento de frecuencia respiratoria, sudoración, tensión muscular, sensaciones gástricas, entre otros. La persistencia en el tiempo de dichos cambios puede traer otro tipo de desórdenes psicofisiológicos tales como, insomnio, dolores de cabeza, náuseas, inapetencia, disfunciones gástricas, entre otros.

Tal como reseñaron los autores, las señales de alerta que surgen de la experiencia subjetiva de la ansiedad, activa mecanismos fisiológicos de acción en el organismo que en condiciones naturales son caracterizadas como normales; lo disfuncional ocurre cuando este tipo de alertas se prolongan en el tiempo y aumentan su intensidad pues el cuerpo se encuentra en un estado perenne de alerta lo que compromete el normal funcionamiento de los diversos sistemas que lo componen.

Otro tipo de manifestación de la ansiedad, expresa Villarroel (2008), son las manifestaciones conductuales, menciona el autor que la ansiedad es un sistema de alerta del organismo ante situaciones consideradas amenazantes, y su función es advertir a la persona mediante la activación del organismo, a fin de movilizarle ante 
situaciones que representan un riesgo no concreto y dar de esta manera la posibilidad de huir. Por lo tanto, las señales o manifestaciones conductuales que se observan son irritabilidad, llanto fácil, sensación de inquietud, sobresaltos o variaciones de humor, depresión, impaciencia, intranquilidad, temblor de manos, fruncimiento del entrecejo, rostro preocupado, falta de placer en los pasatiempos, entre otros.

Sobre lo expuesto por el autor, cabe destacar que las manifestaciones conductuales son muy variadas y que no todas ocurren de la misma manera en las personas, estas suelen ser las señales de mayor notoriedad y que son las percibidas por el círculo social cercano en el cual la persona se desenvuelve. Dichas manifestaciones generan un ambiente disarmónico que afecta las relaciones laborales y compromete la eficiencia personal y del equipo de trabajo; de igual manera entorpece el manejo adecuado de herramientas y actitudes que favorecen el logro de los objetivos de la empresa en concordancia con las políticas corporativas.

Ahora bien, frente a experiencias de gran carga emocional todo ser humano desarrolla mecanismos para dar respuesta, estos mecanismos son conocidos como estilos de afrontamiento los cuales a su vez han sido objeto de estudio en la psicología y son concebidos como mecanismos de adaptación de la persona ante situaciones estresantes o ansiógenas.

En tal sentido, Lazarus (2000) define el afrontamiento como aquellos esfuerzos cognitivos y conductuales, en constante cambio, que son desarrollados para manejar las demandas específicas de atención y afectividad que pueden tener origen externo o interno y que son evaluadas como excedentes o desbordantes de los recursos del individuo.
En concordancia con lo planteado por el autor antes mencionado, se encuentra lo expuesto por Sepúlveda, Romero y Jaramillo (2012), quienes describen al afrontamiento como un esfuerzo cognitivo y conductual dinámico, orientado a manejar, reducir, minimizar, dominar o tolerar dichas demandas externas e internas que generan ansiedad o estrés. El afrontamiento es un aspecto muy relevante del funcionamiento personal y se divide en estilos y estrategias de afrontamiento.

En este sentido, Sepúlveda, Romero y Jaramillo (2012) definen los estilos de afrontamiento como aquellas predisposiciones personales para hacer frente a diversas situaciones que surgen en la cotidianidad, estas suelen ser estables y consistentes según el contexto y es en función de estos, los estilos, que se generan las estrategias.

Según lo planteado por los autores, los estilos de afrontamiento son detonantes de las estrategias de afrontamiento y están ligados a las características de la personalidad, de allí que los factores: carácter, resiliencia, tolerancia, temperamento, entre otros son los agentes propulsores del estilo a implementar.

En cuanto a las estrategias de afrontamiento ya identificadas como esfuerzos o mecanismos conductuales que se ponen en práctica para afrontar las demandas internas o ambientales que se presentan, hay que mencionar que estas conductas, en su intento de restablecer el equilibrio con el ambiente, pueden ser manifiestas o no, de allí que Gantiva, Luna y otros (2010) consideren a las estrategias de afrontamiento como un conjunto de recursos y esfuerzos de orden cognitivo y conductual orientados a resolver el problema, a reducir y a eliminar la respuesta 
emocional o a modificar la evaluación inicial de la situación.

Las estrategias, a diferencia de los estilos de afrontamiento, son procesos concretos y específicos que se utilizan en cada contexto y pueden ser cambiantes dependiendo de las condiciones que las promueven.

En este mismo orden de ideas, Lazarus y Folkman (1986) destacan que las estrategias de afrontamiento se dividen en dos grandes grupos, las centradas en el problema y las centradas en las emociones. Las Estrategias de afrontamiento centradas en el problema se refieren a que la persona se centra en hacer frente a la situación, es decir, son orientadas a la tarea que se debe ejecutar para alcanzar modificar el problema solucionándolo, buscan soluciones al problema que ha provocado la disonancia cognitiva, éstas se refieren a la confrontación, la búsqueda de apoyo social y la búsqueda de soluciones.

Por el contrario, las estrategias de afrontamiento centradas en la emoción, tienden a percibir el evento estresante como incontrolable, se enfocan en la regulación de las consecuencias emocionales activadas por la presencia de la situación estresante mediante el autocontrol, el distanciamiento, la revaluación positiva, la autoinculpación y el escape o evitación.

Generalmente las investigaciones referidas al tema de la ansiedad hacen mención a dos tipos generales de estrategias o escalas de afrontamiento, las cuales según Lazarus y Folkman (1986), son la de resolución de problemas y la de regulación emocional.

De igual manera Sepúlveda, Romero y Jaramillo (2012) clasifican las estrategias de afrontamiento en: estrategias activas referidas a: comportamientos relacionados al problema y estrategias pasivas $\mathrm{o}$ relacionadas con las emociones.

En cuanto a las estrategias activas u orientadas al problema, Rua (2014), las define como aquellas que están orientadas a la modificación de la situación que representa una amenaza para el sujeto, es decir, basado en una deducción costo beneficio operando sobre el sujeto o su entorno, se transforman en una solución que genera alternativas para manejar o minimizar el impacto del problema.

Los autores citados destacan que este tipo de estrategias se caracterizan por la participación activa del individuo y por surgir en situaciones donde se estima tener cierto grado de control, por lo tanto, se hace mayor uso de las herramientas cognitivas y no generan desgaste emocional pues la persona se orienta a la evaluación de una situación, búsqueda de alternativas de solución y administración de los recursos que posee para tal fin poniendo de manifiesto su compromiso hacia el abordaje del problema.

Las estrategias de afrontamiento orientadas a la resolución del problema que serán observadas en esta investigación se describen a continuación según lo citado por Cano, Rodríguez y García (2007):

Resolución de problemas: son estrategias cognitivas y conductuales encaminadas a eliminar el estrés modificando la situación que lo produce. Sandin y Chorot (1993) la definen como la secuencia de acciones orientadas a solucionar el problema, en el momento oportuno.

Reestructuración cognitiva: estrategias cognitivas que modifican el significado de la situación estresante. Dentro de esta perspectiva Cano, Rodríguez y García (ob. cit) aseguran que desviar el significado de la situación estresante hacia los posibles aspectos positivos que tenga o haya tenido 
(fijarse en lo positivo), puede aminorar el impacto emocional de la situación estresante.

Apoyo Social: estrategia referida a la búsqueda de apoyo emocional. Según lo expuesto por Cano, Rodríguez y García (ob. cit) esta estrategia está encaminada a la búsqueda de apoyo, a liberar las emociones que acontecen en el proceso de estrés.

Expresión emocional: estrategia encaminada a liberar las emociones que acontecen en el proceso de estrés. Por su parte Cano, Rodríguez y García (ob. cit) señalan que en esta estrategia el sujeto está convencido de lo estresante de la situación, pero también de la posibilidad que tiene de ser modificada, convicción que le permite centrar su esfuerzo en apaciguar el malestar emocional que pueda generarse.

En relación con las estrategias pasivas u orientadas a la emoción, Rua (2014), explica que las mismas no están destinadas a la modificación o manejo de la situación, pues suelen existir situaciones no modificables en el momento o de manera permanente, por lo tanto, las acciones que se tomen son orientadas más a la autoprotección que a la resolución de un problema pues se evalúa el nivel de compromiso que implica la situación ansiógena ya que su carga emocional es alta y lo que se busca es tolerarla.

Al respecto, Jorques (2015), describe este tipo de estrategias como aquellas que están dirigidas a controlar la repercusión emocional provocada por la situación estresante; para ello la persona desarrolla diferentes acciones: comportamentales, distractoras, sociales, o cognitivas como la reestructuración de la situación estresante o la negación de la misma. El propósito final de dichas estrategias es modificar la interpretación de las emociones, mediante la selección de aspectos a los que prestar más atención, o bien alterando su significado.
Este tipo de afrontamiento aparece cuando la persona se siente sin control sobre la situación amenazante.

Según lo planteado por los autores se puede inferir que las estrategias orientadas a la emoción son de carácter individual y no requieren recursos externos para ser empleadas, por lo tanto el objetivo final de las mismas no es modificar una situación si no actuar sobre la interpretación de la misma y minimizar así, en la medida de lo posible, su impacto emocional, es decir, actúa más como mecanismo de defensa que como participación activa para solventar un problema.

Como bien explican los autores, el no asumir los problemas y evitarlos, conlleva a poseer pensamientos irracionales lo cual afecta de diversas formas, es así como el manejo inadecuado de una estrategia puede acarrear manifestaciones patológicas que comprometen el bienestar del individuo y su medio social.

En tal sentido, la selección de la estrategia a utilizar depende de la valoración que se otorgue a la situación, lo cual está sujeto a la disponibilidad de recursos, que pueden ser de origen individual o ambiental. Se infiere entonces que el principal propósito de la estrategia es, valiéndose de cualquier tipo de esfuerzo, servir de herramienta adaptativa que permita mitigar o manejar una situación ansiógena o estresante, teniendo en cuenta que cada sujeto tiende, en una situación de incertidumbre o generadora de ansiedad, a la utilización de las estrategias de afrontamiento que domina por aprendizaje o por hallazgo fortuito.

De igual manera la presente investigación tiene apoyo o fundamentación legal en diferentes documentos que resaltan la protección física y social de las personas en el área laboral. 
En efecto, la Constitución de la República Bolivariana de Venezuela, de 1999 establece que toda persona tiene derecho al trabajo, por otra parte garantiza los derechos de los ciudadanos como trabajadores y se vela porque tengan un ambiente apto, y un trato digno y justo.

Artículo 3. El Estado tiene como fines esenciales, la defensa y el desarrollo de la persona y el respeto a su dignidad, el ejercicio democrático de la voluntad popular, la construcción de una sociedad justa y amante de la paz... La educación y el trabajo son los procesos fundamentales para alcanzar dichos fines. (p. 4).

Es evidente que la carta magna establece desde sus primeros artículos, postulados y ordenanzas, el favorecer la evolución de los seres humanos en cuanto al desenvolvimiento en sus diversas actividades, por ello es indispensable, bajo el enfoque organizacional, que los patronos, y las líneas de mando de las mismas, se esmeren por aportar las mejores condiciones laborales en todos los sentidos a las personas, o sea que éstas se desarrollen en los diferentes ámbitos de los sectores donde hacen vida activa, en condiciones idóneas que les permitan adaptarse al entorno y alcanzar las metas propuestas.

Artículo 87. Toda persona tiene derecho al trabajo y el deber de trabajar. El Estado garantizará la adopción de las medidas necesarias a los fines de que toda persona puede obtener ocupación productiva, que le proporcione una existencia digna y decorosa y le garantice el pleno ejercicio de este derecho. Es fin del Estado fomentar el empleo. La ley adoptará medidas tendentes a garantizar el ejercicio de los derechos laborales de los trabajadores y trabajadoras no dependientes. La libertad de trabajo no será sometida a otras restricciones que las que la ley establezca. ( $\mathrm{s} / \mathrm{p})$.
Por ser esta investigación de corte clínico, y no obstante su indagación es en la temática laboral, el artículo anteriormente mencionado es de gran relevancia ya que confirma la seguridad de trabajo que brinda el estado a todos los venezolanos y al mismo tiempo ampara sus derechos, lo que garantiza el bienestar de los trabajadores.

Asimismo, la Ley orgánica de prevención de condiciones y medio ambiente del trabajo (LOPCYMAT, (2005) en sus artículos 10 y 12 señala la importancia de la vigilancia del ambiente y de las condiciones de trabajo, entendiendo por estas, "aquellas condiciones generales y especiales, bajo las cuales realiza la ejecución de la tareas y los aspectos organizativos de las empresas y empleadores". (p. 21). Esta ley asegura a las/los trabajadores, el disfrute de un estado de salud física y mental normal y la protección adecuada a la mujer, y a las personas naturales en condiciones especiales.

Otro documento legal que menciona la protección física y social del individuo es la Ley del ejercicio de la psicología la cual en su artículo dos (2) establece que el ejercicio de la psicología tiene como fin contribuir a la prevención y modificación de aquellas conductas que afecten la evolución psicológica normal del individuo.

Artículo 2...Igualmente lo capacita para contribuir en la prevención de las dificultades de la evolución psicológica normal del individuo; para la elaboración de programas que favorezcan el desarrollo personal, educativo y social del hombre, y para la solución de problemas en la conducta mediante el empleo de técnicas y procedimientos psicológicos. 
Como puede observarse en la mencionada ley, es válido desarrollar técnicas 0 actividades investigativas orientadas al desarrollo personal, educativo y social del hombre, ajustándose a los principios de salud física y mental como lo establece la constitución nacional respecto a garantizar el mantenimiento de la dignidad humana del ciudadano.

De igual manera, el código de ética profesional del psicólogo en su capítulo 1 toma en consideración los artículos 55 y 60 para el desarrollo de esta investigación en los cuales se establece como condición para una investigación de corte psicológico que sea realizada y su pervisada por personas técnicamente entrenadas y científicamente calificadas, asi como que se garantice la debida protección de las respuestas de los sujetos sometidos a investigación para evitar así que se establezca trazabilidad alguna que pueda ocasionar daños morales.
MÉTODO

Esta investigación, estuvo enmarcada dentro de un enfoque cuantitativo positivista-de tipo correlacional. La población de estudio estuvo conformada por aproximadamente 40 sujetos compuesto por trabajadores contemplados como mano de obra directa e indirecta de la empresa EHCOPEK-S.A. ubicada en Maracaibo, estado Zulia.

Para la recopilación de datos referentes a las manifestaciones de la ansiedad y las estrategias de afrontamiento se utilizó la técnica de la encuesta y como instrumento para la realización del estudio se utilizó: el Inventario de Ansiedad Rasgo - Estado de Spielberg (IDARE) (1975): La escala Ansiedad rasgo (SXR). La escala de ansiedad estado (SXE) La corrección se realizó con plantillas que otorgan el valor numérico adecuado y la sumatoria total indicó la calificación por escala, calificándose según la siguiente estructura:

Cuadro 1. Niveles de ansiedad de test IDARE

\begin{tabular}{cl}
\hline Rango de puntuación & Clasificación de ansiedad \\
\hline $20-31$ & Ansiedad muy baja \\
$32-43$ & Ansiedad baja \\
$44-55$ & Ansiedad media \\
$56-67$ & Ansiedad alta \\
$68-80$ & Ansiedad muy alta \\
\hline
\end{tabular}

Fuente: Spielberg (1975)

Otro de los instrumentos utilizados para evaluar las estrategias de afrontamiento, fue el Inventario de Estrategias de Afrontamiento (CSI), adaptado por Cano, Rodríguez y García (2007).

El inventario, posee un formato tipo Likert de cinco (5) opciones de repuesta para su interpretación, las puntuaciones se obtienen sumando el valor de las respuestas semejantes de un ítem en una escala específica y dividiéndola entre el número de ítems para arrojar el grado de uso de la estrategia de afrontamiento tal como se muestra a continuación: 
Cuadro 2. Baremo interpretación de inventario de estrategias de afrontamiento (CSI)

\begin{tabular}{cc}
\hline Valor & Uso de la estrategia \\
\hline 0 & No, en lo absoluto \\
1 & Un poco \\
2 & Bastante \\
3 & Mucho \\
4 & Totalmente \\
\hline
\end{tabular}

Fuente: Cano, Rodríguez y García (2007)

En tal sentido, la confiabilidad del Inventario de Ansiedad Rasgo - Estado (IDARE) se obtuvo a través del método test retest en el que se obtuvieron puntuaciones para la escala SXR de 0.73 a 0.86 y para la escala SXE, dada la naturaleza transitoria de los estados de ansiedad, para las medidas de consistencia interna se obtuvo un coeficiente alpha de cronbach cuyo valores oscilaron entre 0.83 a 0.92 , lo que muestra que la consistencia interna o confiabilidad de ambas escalas es alta.

En referencia a la confiabilidad del instrumento Inventario de Estrategias de Afrontamiento (CSI), los resultados se obtuvieron por medio del cálculo alfa de Cronbach, arrojando puntajes altos $(0,914)$ para la escala global, lo que demuestra que el instrumento posee longitud y 0.63 a 0.86 para las estrategias de afrontamiento de la escala, lo cual señala que el inventario es homogéneo con respecto al constructo medido y sus puntajes son consistentes dentro de la muestra. En cuanto al análisis de los datos que se obtuvieron de la aplicación de los instrumentos de recolección de datos, éste se realizó mediante la estadística descriptiva de frecuencia absoluta y porcentual para lo cual se utilizó el paquete estadístico para las Ciencias Sociales (SPSS), versión 20 y Microsoft Excel 2010.

\section{RESULTADOS}

En el cuadro 3, se observan los datos estadísticos de la variable ansiedad según la dimensión ansiedad estado, el test arroja valores que oscilan de 20 a 80 puntos, lo que indica que entre más alto es el puntaje será mayor el nivel de ansiedad. El comportamiento de la muestra revela que en el nivel de ansiedad estado existe un mínimo de 44 y un máximo de 63 puntos respectivamente; de igual manera se evidencia que la media se ubicó en 55,55 puntos y una desviación típica de 5,368 con una dispersión alta, indicando que está distribuida de manera heterogénea de los resultados con relación a la muestra, para tal instrumento según el baremo de interpretación se encuentra ubicado en una ansiedad media, pero una muestra menor indica que posee una baja ansiedad estado. Es decir, que la mayoría de personas encuestadas presenta niveles de ansiedad estado medianamente significativos y una minoría, pero que genera relevancia, se ubican en nivel bajo.

Sobre este punto, Spielberg (1972), la define la ansiedad estado como un estado emocional inmediato y modificable en el tiempo, que se caracteriza por una combinación única de sentimientos de tensión, aprensión, nerviosismo, pensamientos molestos y cambios fisiológicos. Cabe destacar que la ansiedad 
estado no se manifiesta directamente en la conducta y debe ser inferida por la frecuencia con la que un individuo experimenta aumentos en sus estados de ansiedad. Los niveles altos de la misma son apreciados como molestos y obligan al individuo a poner en marcha habilidades que le permitan afrontar la situación causante de la aparición de la misma.

En el caso de la muestra de los trabajadores petroleros de la empresa EHCOPEK,S.A, éstos se encuentran en constante ansiedad estado, es decir, constantemente se encuentran en vicisitudes que los alerta y lleva a situaciones de malestar, nerviosismo, por lo que sus funciones fisiológicas se alteran, lo que indica que probabilísticamente pueden estar propensos a desencadenar respuestas defensivas cuyas manifestaciones pueden darse a nivel cognitivo, conductual y fisiológicos con posibilidad de ser evidenciadas a través de cambios temporales en la persona que pueden ser dañinos para la salud psicosocial del trabajador. La persistencia en el tiempo de dichos cambios puede traer otro tipo de desórdenes psicofisiológicos tales como, insomnio, dolores de cabeza, náuseas, inapetencia, disfunciones gástricas, entre otros que pueden comprometer realmente su estabilidad laboral.

Cuadro 3. Estadísticos descriptivos de la variable ansiedad según la dimensión ansiedad estado

\begin{tabular}{ccc}
\hline ANSIEDAD & Estado & Nivel \\
\hline Media & 55,55 & \\
Desv. típ. & 5,368 & \\
Asimetría &,- 737 & Media \\
Curtosis &,- 647 & \\
Mínimo & 44 & \\
Máximo & 63 & \\
\hline
\end{tabular}

En el cuadro 4, se observan los datos estadísticos de la variable ansiedad según la dimensión ansiedad rasgo, el test arroja un valor que oscilan de 20 a 80 puntos, lo que indica que entre más alto es el puntaje será mayor el nivel de ansiedad. El comportamiento de la muestra revela que en el nivel de ansiedad rasgo existe un mínimo de 27 y máximo 63 puntos respectivamente; de igual manera se evidencia que la media se ubicó en 53,33 puntos y una desviación típica de 6,761 con una dispersión baja, lo cual muestra que está distribuida de manera homogénea de los resultados con relación a la muestra, para tal instrumento el resultado según el baremo de interpretación se encuentra ubicado en un nivel de ansiedad media. Es decir, la mayoría de personas encuestadas presenta niveles de ansiedad rasgos medianamente significativos.

En este sentido, el autor Spielberg (1972), explica que la ansiedad rasgo hace referencia a las diferencias individuales de ansiedad relativamente estables, siendo éstas una disposición, tendencia o rasgo, por lo tanto, aquello sujetos con alto grado de ansiedad rasgo son más propensos a experimentar alto grado de ansiedad estado de manera más frecuente; en otras palabras, la ansiedad rasgo se encuentra inmersa 
dentro de las características de la personalidad y por lo tanto los individuos que incluyen este tipo de ansiedad ven con mayor peligrosidad aquellas situaciones que les resulten amenazantes, por lo que desarrollan estrategias de afrontamiento más rápidas y más agresivas.

En el caso de los trabajadores objeto de este estudio, un nivel medio de ansiedad rasgo permite inferir que la mayoría consideran estar en situaciones amenazantes o responden ante éstas con niveles medios de ansiedad, por lo que constantemente, en la mayoría de las situaciones, presentan características de ansiedad que les genera inestabilidad significativa.

Cuadro 4. Estadísticos descriptivos de la variable ansiedad según la dimensión rasgo

\begin{tabular}{ccc}
\hline ANSIEDAD & Estado & Nivel \\
\hline Media & 53,33 & \\
Desv. típ. & 6,761 & \\
Asimetría & $-1,338$ & Media \\
Curtosis & 4,636 & \\
Mínimo & 27 & \\
\hline
\end{tabular}

En cuanto a los resultados de la aplicación del instrumento para medir la Variable de Estrategias de afrontamiento y sus dimensiones para dar así respuesta al objetivo de identificar las estrategias de afrontamiento empleadas por los trabajadores petroleros de la empresa EHCOPEK-S.A. ubicada en el estado Zulia, el cuadro 5 exhibe el comportamiento de los resultados en cuanto a la Variable Estrategias de Afrontamiento según la dimensión activa u orientada al problema, en el cual se muestra una puntuación máximo 4 y como mínimo 1 con una media de 2,05 puntos y una desviación típica de 0,749 que refleja una dispersión baja en los resultados en función a la media, poniendo de manifiesto una muestra heterogénea, que revela que las estrategias de afrontamiento más utilizadas son las activas $\mathrm{u}$ orientadas al problema.

Este resultado coincide con lo expresado por Rua (2014), quien indica que las estrategias activas $u$ orientadas al problemas son las orientadas a la modificación de la situación que representa una amenaza para el sujeto, es decir, se transforman en una solución que genera alternativas para poder manejar o minimizar el impacto del problema basado en una deducción costobeneficio que opera sobre el sujeto o su entorno. Al mismo tiempo este resultado indica que los trabajadores en estudio tienen cierto control sobre la situación, e intentan buscar equilibrio entre el agente estresor y su adaptación física y psíquica. 
Cuadro 5. Estadísticos descriptivos de la variable estrategias de afrontamiento según la dimensión activa u orientada al problema

\begin{tabular}{cc}
\hline Categoría & Activas u orientadas al problema \\
\hline Media & 2,05 \\
Desv. típ. &, 749 \\
Asimetría &, 302 \\
Curtosis &,- 141 \\
Mínimo & 1 \\
Máximo & 4 \\
\hline
\end{tabular}

De igual manera, el cuadro 6, muestra el comportamiento de los resultados de la Variable Estrategias de Afrontamiento según la dimensión pasiva u orientada a la emoción, donde se obtuvo una puntuación máximo de 3 y mínimo de 1 , con una media de 1,35 puntos y una desviación típica de 0,580 que refleja una dispersión alta en los resultados en función a la media, evidencia una muestra heterogénea, y revela que son poco utilizadas las estrategias de afrontamiento pasivas $\mathrm{u}$ orientadas a la emoción.

En relación a las estrategias pasivas u orientadas a la emoción Rua (2014), explica que no están destinadas a la modificación o manejo de la situación, pues suelen existir situaciones inmodificables en el momento o de manera permanente, por lo tanto, las acciones que se tomen son orientadas más a autoprotección que a la resolución de un problema pues se evalúa el nivel de compromiso que implica la situación ansiógena ya que su carga emocional es alta y lo que se busca es poder tolerarla.

El resultado obtenido en el estudio en cuanto a la utilización de estrategias de afrontamiento pasivas $\mathrm{u}$ orientadas a la emoción están acordes con lo planteado por el autor mencionado y permite inferir que la muestra de trabajadores de la empresa en estudio, a pesar de tener situaciones estresantes, desarrollan diferentes acciones comportamentales, para afrontar directamente el problema sin detenerse en distractores sociales o cognitivos, sino que por el contrario reconocen el problema existente y buscan su solución; por lo que poco utilizan la estrategia de afrontamiento pasivo.

Cuadro 6. Estadísticos descriptivos de la variable estrategias de afrontamiento según la dimensión pasiva u orientada a la emoción

\begin{tabular}{cc}
\hline Categoría & Pasivas u orientadas a la emoción \\
\hline Media & 1,35 \\
Desv. típ. &, 580 \\
Asimetría & 1,460 \\
Curtosis & 1,269 \\
Mínimo & 1 \\
\hline
\end{tabular}




\section{CONCLUSIONES}

Se establece que los trabajadores petroleros de la empresa EHCOPEK-S.A ubicada en el estado Zulia, presentan niveles de ansiedad estado en un nivel medio, es decir, la mayoría de los sujetos se encuentran en una situación medianamente amenazante, lo cual es propicio para el desarrollo de manifestaciones somáticas y la puesta en marcha de mecanismos de afrontamiento. Sin embargo, una muestra menor presenta ansiedad estado en bajo nivel con una tendencia a nivel medio.

En lo que respecta a la ansiedad rasgo, se evidencian niveles medios en la mayoría de los encuestados. Se considera que existe una alta propensión a desarrollar rápidamente estados de ansiedad en situaciones amenazantes, por ser esta una característica de la personalidad, lo que aunado a la exposición constante a situaciones de incertidumbre o incomodidad puede generar patologías de orden fisiológico y emocional.

En relación a la generación de estrategias de afrontamiento que poseen los trabajadores petroleros de la mencionada empresa se evidencia que la mayoría (aproximadamente el $78 \%$ de la muestra) utiliza estrategias activas u orientadas a la solución del problema, lo que se considera positivo para el bienestar psicológico de la persona.

De igual manera se observa que son poco usadas las estrategias pasivas $u$ orientadas a la emoción pues los trabajadores demuestran comprensión de la situación amenazante y por ende entienden la necesidad de generar soluciones, por lo que consideran que el valerse de estrategias pasivas no constituye una herramienta útil para el contexto descrito

Los resultados obtenidos permiten concluir que no existe una relación entre la ansiedad y las estrategias de afrontamiento presentes en los trabajadores de EHCOPEK S.A. ya que cada variable actúa de manera independiente, presentándose niveles medios de ansiedad con estrategias de afrontamiento orientadas en la solución del problema, razón por la cual las situaciones amenazantes son abordadas de manera activa y la persona idea mecanismos sanos de defensa para hacer frente a la misma y lograr una adecuada adaptabilidad en el contexto que se desenvuelve.

Factores como la edad y la experiencia laboral de larga data en este tipo de trabajos han permitido el empoderamiento de los trabajadores lo que les sirve de herramienta de apoyo al presentarse este tipo de eventos.

\section{REFERENCIAS}

Asociación Americana de Psiquiatría (2014). Guía de consulta de los criterios diagnósticos del DSM-5. Arlington

Cano, F. Rodríguez, L. García, J. (2007). Adaptación española del Inventario de Estrategias de Afrontamiento. Disponible:

http://personal.us.es/fjcano/drupal/file s/AEDP\%2007\%20(esp).pdf [Consultado: 2018 Noviembre, 14]

Cano, M. (1986). ISRA: Manual del inventario de situaciones y respuestas de ansiedad. TEA Ediciones. Madrid

Charry, A. (2012). Sobre la ansiedad y estrategias de afrontamiento en relación a la competencia deportiva en el atletismo. Una revisión de temas primordiales. Universidad del Valle. Cali

Código de ética del Psicólogo (1981). Federación de Psicólogos de Venezuela

Constitución de la República Bolivariana de Venezuela (1999). Transcripción en línea disponible en: (Consulta: 2016, octubre 5)

Cooper, C. Payne, R. (1992). International perspectives on research into work, wellbeing and stress management. 
Enciclopedia de Salud y Seguridad en el trabajo. Washington, DC: APA Press

Gantiva, C. Luna, A. Dávila, A. Saalgado, M. (2010). Estrategias de afrontamiento en personas con ansiedad. Psychologia. Avances de la disciplina, vol. 4, núm. 1, enero-junio, 2010, pp. 63-72 Universidad de San Buenaventura. Bogotá

Jorques, M. (2015). Estrategias de afrontamiento ante el cáncer de mama. Universidad de Valencia. Valencia

Kort, F. García, J. Pérez, L. (1998). Estado Psicológico del Habitante de Caracas (Venezuela). Revista Latinoamericana de Psicología, 30 (1): 137- 146

Lazarus, R. (2000). Estrés y emoción: Manejo $e$ implicaciones en nuestra salud. Editorial Desclée de Brouwer, S.A. Bilbao

Lazarus, R. y Folkman, S. (1986): Estrés y procesos cognitivos. Barcelona: Ediciones Martínez Roca. Disponible: http://www.moldesmentales.com/otros /mar.htm [Consulta: 2018, octubre 2]

Ley del ejercicio de la Psicología (1978). Gaceta oficial de la República de Venezuela año CV- mes XII nro. 2.306. Caracas

Ley orgánica de prevención, condiciones y medioambiente de trabajo (2005). Gaceta oficial de la República Bolivariana de Venezuela

Lluch, M., Novel, G. y Ortiz, A. (2005) La Ansiedad y el Estrés como: Componentes básicos del enfermar. Enfermería Psicosocial y Salud Mental. Ed. Masson; 2005 p. 165 -173. España

Medrano, L. Mirantes, R. (2008). Manifestaciones cognitivas de ansiedad y su relación con el rendimiento académico. Jornadas de Investigación y Cuarto Encuentro de Investigadores en Psicología del Mercosur. Buenos Aires
Navarrete, M. (2013). La ansiedad: ¿Cómo nos afecta? Disponible: http://www.guiadelasalud.info/articulo. php?art=638\&id=480 [Consulta: 2019, Febrero 2]

Organización Mundial de la Salud (2008). Sensibilizando sobre el estrés laboral en los países en desarrollo. Serie nro. 6. Francia

Rua, M. (2014). Ansiedad y estilos de afrontamiento en pacientes femeninos con diabetes mellitus tipo 1 y 2 . Universidad Rafael Urdaneta. Maracaibo Sandin, V. y Chorot, P. (1993). Stress and Anxiety: Diagnosis Validity of Anxiety Disorders According to life events stress, Ways of coping and physical symptoms. Psiquis, 14, 178-184

Sepúlveda, A. Romero, A. y Jaramillo, L. (2012). Estrategias de afrontamiento y su relación con depresión y ansiedad en residentes de pediatría en un hospital de tercer nivel. Boletín médico del Hospital Infantil de México, 69(5), 347-354. Disponible:

http://www.scielo.org.mx/scielo.php?sc ript=sci_arttext\&pid=S1665-

$11462012000500005 \&$ Ing=es\&tlng=es. [Consulta: 2019, febrero 17]

Sociedad Española de Medicina Psicosomática. (2016). Contexto actual de los problemas de ansiedad en el entorno laboral. XLIV Congreso de la Sociedad Española de Medicina Psicosomática. Madrid

Spielberg, C. (1972). Ansiedad como un estado emocional. México: Tendencias corrientes en la teoría e investigación

Villarroel, J. (2008). Causas de la Ansiedad: Origen y Mantenimiento. Disponible:http://www.clinicadeansieda d.como/02/257/Causas-de-la-ansiedad:origen-y-mantenimiento.htm. [Consulta: 2018, septiembre 29] 